\title{
On the orientational dependence of giant magnetoresistance
}

\author{
C. Blaas ${ }^{1,2}$, P. Weinberger ${ }^{1,2}$, L. Szunyogh ${ }^{2,3}$, J. Kudrnovský2 ${ }^{2,4}$, V. Drchal ${ }^{2,4}$, P. M. Levy ${ }^{5}$, and C. Sommers ${ }^{6, a}$ \\ 1 Institut für Technische Elektrochemie, Technische Universität Wien, Getreidemarkt 9/158, 1060 Wien, Austria \\ 2 Center for Computational Materials Science, Gumpendorferstrasse 1a, 1060 Wien, Austria \\ 3 Department of Theoretical Physics, Technical University Budapest, Budafoki út 8, 1521 Budapest, Hungary \\ 4 Institute of Physics, Czech Academy of Sciences, 18040 Praha 8, Czech Republic \\ ${ }^{5}$ Department of Physics, New York University, New York, NY 10003, USA \\ ${ }^{6}$ Laboratoire de Physique des Solides, Université de Paris-Sud, 91405 Orsay Cedex, France
}

Received 30 November 1998

\begin{abstract}
The functional dependence of the giant magnetoresistance (GMR) with respect to the relative angle between the orientations of the magnetization in the magnetic slabs of a trilayer system is calculated by using the Kubo-Greenwood formula for electrical transport together with the fully-relativistic spinpolarized screened Korringa-Kohn-Rostoker method for semi-infinite systems and the coherent potential approximation. It is found that the functional dependence of the GMR is essentially of the form $(1-\cos \varphi)$.
\end{abstract}

PACS. 71.20.Be Transition metals and alloys $-72.15-\mathrm{v}$ Electronic conduction in metals and alloys 75.30.Et Exchange and superexchange interactions

\section{Introduction}

One of the outstanding unresolved questions about the giant magnetoresistance (GMR) of magnetic multilayers is its functional dependence with respect to the relative angle between the orientations of the magnetization in the magnetic slabs. Some results have been obtained for free electron models when only spin-dependent scattering has been considered. These results show that there is a simple $\cos \varphi$ dependence in the limit when the mean free path of the electrons is large compared to the thickness of the layers [1]. When the role of the variation of the lattice potential from layer to layer is included in the free electron models, one finds deviations from the $\cos \varphi$ dependence [2], but no clear trends have been established. For example, Vedyayev et al. (see Ref. [2]) discussed the general case with both spin-dependent scattering and potential barriers between successive layers for a spin valve structure, and showed that when the spin-dependent lattice potential is included, the resistivity is no longer a linear function of $\cos \varphi$. For current in the plane of the layers (CIP), experiments have found for the most part, the simple $\cos \varphi$ behavior [3]; however for current perpendicular to the plane of the layers (CPP), significant deviations have been established [4]. What is lacking up till now is a calculation of the orientational dependence of GMR that at the same time is based on realistic band structures and is able to account for spin-dependent scattering.

We have developed the means for calculating the GMR of multilayered structures that incorporates both

\footnotetext{
a e-mail: sommers@lps.u-psud.fr
}

electronic structure and scattering [5]. We use a fully-relativistic spin-polarized screened Korringa-KohnRostoker (SKKR) method for the electronic structure of layered systems, the coherent potential approximation (CPA) for the scattering, and the Kubo-Greenwood formula for the conductivity. To date, we have considered only collinear magnetic configurations of the magnetic slabs, i.e., parallel and antiparallel; here we determine the complete variation of the resistivity with angle, and thereby the angular variation of GMR. As our method is fully relativistic, it includes spin orbit coupling which relates the orientation of the magnetization to the lattice; therefore, we are able to assess the extent to which this influences resistivity and GMR. In addition, we have previously used the same SKKR method to calculate the interlayer exchange coupling (IEC) of multilayered structures, and it is of interest to compare the angular dependence of the GMR and IEC. There have been suggestions that the two are related for the CPP geometry [6], but for CIP there is no reason for similar orientational dependencies for the GMR and the IEC.

Here we present the results on the functional dependence of resistivity with respect to the relative angle between the orientations of the magnetization in the magnetic slabs. As we have only determined resistivity for CIP, we limit our considerations to this geometry. In this first attempt, the simplest possible case of a magnetic trilayer system is chosen such that by varying the orientation of the magnetization in one of the magnetic slabs, one can see the resistivity of the system changing with respect to the ferromagnetic (reference) configuration. To see the 
influence of the orientation of the magnetization relative to the plane of the layers, we have determined the angular variation of the resistivity and GMR for magnetization in and out of the plane of the layers. The orientational dependence of IEC for the same systems will be compared to that of the GMR to determine what, if any, similarities exist for CIP.

\section{Theoretical approach}

In the Kubo-Greenwood approach, the $\mu \nu$ th element of the electrical conductivity tensor is given by (for a review, see, e.g., Ref. [7]):

$$
\sigma_{\mu \nu}=\frac{\hbar}{\pi \Omega} \operatorname{Tr}\left\langle J_{\mu} \operatorname{Im} G^{+}\left(\epsilon_{\mathrm{F}}\right) J_{\nu} \operatorname{Im} G^{+}\left(\epsilon_{\mathrm{F}}\right)\right\rangle .
$$

In this equation $\mu, \nu \in\{x, y, z\}, \Omega$ is the volume, $J_{\mu}$ is the $\mu$ th component of the current operator, $\epsilon_{\mathrm{F}}$ is the Fermi energy, and $\langle\cdots\rangle$ denotes an average over configurations.

In general, in a relativistic spin-polarized multiple scattering theory, the Green's function $G\left(\mathbf{r}, \mathbf{r}^{\prime}, z\right)$ is described via an effective Kohn-Sham-Dirac Hamiltonian [8]:

$$
\mathcal{H}(\mathbf{r})=c \boldsymbol{\alpha} \cdot \mathbf{p}+\beta m c^{2}+I_{4} V(\mathbf{r})+\beta \boldsymbol{\Sigma} \cdot \mathbf{B}^{\mathrm{eff}}(\mathbf{r}),
$$

where the $\boldsymbol{\alpha}_{i}(i=1,2,3)$ and $\beta$ are Dirac matrices, $I_{4}$ is a $4 \times 4$ unit matrix, $\mathbf{p}$ the momentum operator, $m c^{2}$ the rest energy of an electron, $V(\mathbf{r})$ the potential, $\boldsymbol{\Sigma}$ the spin operator, and $\mathbf{B}^{\mathrm{eff}}(\mathbf{r})$ is the (effective) exchange field:

$$
\mathbf{B}^{\mathrm{eff}}(\mathbf{r})=B^{\mathrm{eff}}(\mathbf{r}) \widehat{\mathbf{m}}
$$

with $\widehat{\mathbf{m}}$ being a pre-chosen direction [9]. In equations $(2,3)$ $V(\mathbf{r})$ and $B^{\text {eff }}(\mathbf{r})$ are of muffin-tin form (with $S_{i}$ denoting the radius of the $i$ th muffin-tin sphere):

$$
\begin{gathered}
V(\mathbf{r})=\sum_{i} V_{i}\left(\mathbf{r}_{i}\right) ; \quad V_{i}\left(\mathbf{r}_{i}\right)= \begin{cases}V_{i}\left(r_{i}\right) ; & r_{i} \leq S_{i} \\
\text { const. } ; & \text { otherwise }\end{cases} \\
B^{\mathrm{eff}}(\mathbf{r})=\sum_{i} B_{i}^{\mathrm{eff}}\left(\mathbf{r}_{i}\right) ; B_{i}^{\mathrm{eff}}\left(\mathbf{r}_{i}\right)=\left\{\begin{array}{ll}
B_{i}^{\mathrm{eff}}\left(r_{i}\right) ; & r_{i} \leq S_{i} \\
\text { const. } ; & \text { otherwise }
\end{array},\right.
\end{gathered}
$$

such that in a ferromagnetic reference configuration

$$
\mathbf{B}_{i}^{\mathrm{eff}}\left(\mathbf{r}_{i}\right)=B_{i}^{\mathrm{eff}}\left(\mathbf{r}_{i}\right) \widehat{\mathbf{m}}, \forall i
$$

In a multiple scattering approach based on equation (2), the Kubo-Greenwood formula (Eq. (1)) can be applied on an $a b$ initio level to semi-infinite systems [10], i.e., to systems characterized by only two-dimensional translational symmetry as is the case in magnetic multilayer systems. Our first calculations of the GMR (see Ref. [5]) showed that presently up to a thickness of almost 200 a.u. we obtained reasonable values of the GMR as well as realistic resistivities for magnetic multilayers. Furthermore, within a generalized inhomogeneous CPA scheme we are able to treat the diffuse scattering arising in magnetic multilayers by alloying the layers and by including the effects of the interdiffused interfaces.

Up-to-now, only a comparison between the conductivity of ferromagnetically and antiferromagnetically ordered multilayer systems has been considered in all the $a b$ initio calculations of electrical transport in magnetic multilayer systems: supercell or not. In terms of a spin-polarized fully-relativistic approach, the conductivity tensor can be calculated for non-collinear arrangements of the magnetic slabs; therefore, the GMR can be followed as a function of rotation angle as one moves from a ferromagnetic to the corresponding antiferromagnetic configuration.

We rotate the magnetization of one of the magnetic slabs of the trilayer around an axis $\widehat{\mathbf{n}}$ by an angle $\varphi$, and write the resistivity as

$$
\rho_{x x}(\widehat{\mathbf{n}}, \varphi)=\sigma_{x x}^{-1}(\widehat{\mathbf{n}}, \varphi), \quad \text { etc. }
$$

and the corresponding giant magnetoresistance (GMR) is

$$
\operatorname{GMR}(\widehat{\mathbf{n}}, \varphi)=\frac{\rho_{x x}(\widehat{\mathbf{n}}, \varphi)-\rho_{x x}(\widehat{\mathbf{n}}, 0)}{\rho_{x x}(\widehat{\mathbf{n}}, \varphi)},
$$

where $(\widehat{\mathbf{n}}, 0)$ refers to a pre-chosen magnetic reference configuration, i.e., ferromagnetic configuration.

A function $f(\widehat{\mathbf{n}}, \varphi)$ can be fit by using the following expansion traditionally used for the magnetic anisotropy energy, see, e.g., reference [11],

$$
f(\widehat{\mathbf{n}}, \varphi)=f(\widehat{\mathbf{n}}, 0)+\sum_{m=1}^{\infty} a_{m}(\widehat{\mathbf{n}})\left(1-\cos ^{m} \varphi\right),
$$

where the first two fitting coefficients can be approximated to first order by

$$
\begin{gathered}
a_{1}(\widehat{\mathbf{n}})=\frac{1}{2}[f(\widehat{\mathbf{n}}, \pi)-f(\widehat{\mathbf{n}}, 0)], \\
a_{2}(\widehat{\mathbf{n}})=f\left(\widehat{\mathbf{n}}, \frac{\pi}{2}\right)-\frac{1}{2}[f(\widehat{\mathbf{n}}, \pi)+f(\widehat{\mathbf{n}}, 0)] .
\end{gathered}
$$

We will be interested in ascertaining the difference in resistivity, if any, on rotating the magnetization, in as compared to out of, the plane of the layers. Therefore, we denote rotation out of the plane as a rotation around the $\widehat{\mathbf{y}}$-axis in the plane of the layers by an angle $\Theta$, i.e., a rotation from $\hat{\mathbf{z}}(\Theta=0)$ to $-\hat{\mathbf{z}}(\Theta=\pi)$ (for both the magnetization is perpendicular to the layers), and a rotation in the plane by a rotation around the $\widehat{\mathbf{z}}$-axis perpendicular to the layers by an angle $\Phi$, i.e., a rotation from $\hat{\mathbf{x}}(\Phi=0)$ to $-\hat{\mathbf{x}}(\Phi=\pi)$, in equation $(8)$. In this manner $f(\widehat{\mathbf{y}}, \vartheta)$ can be abbreviated simply by $f(\Theta)$, and $f(\widehat{\mathbf{z}}, \varphi)$ by $f(\Phi)$. This is the notation we use in the following section. It should be recalled that such rotations refer to transformations of the magnetization and only via the scalar product $\boldsymbol{\Sigma} \cdot \mathbf{B}$ in equation (2) induce a transformation of $\boldsymbol{\Sigma}$.

Using the above notation, the interlayer exchange energy is defined as

$$
\operatorname{IEC}(\widehat{\mathbf{n}}, \varphi)=E_{\mathrm{t}}(\widehat{\mathbf{n}}, \varphi)-E_{\mathrm{t}}(\widehat{\mathbf{n}}, 0) \sim E_{\mathrm{b}}(\widehat{\mathbf{n}}, \varphi)-E_{\mathrm{b}}(\widehat{\mathbf{n}}, 0) .
$$


Table 1. Interdiffused trilayers.

\begin{tabular}{|c|c|}
\hline $\mathrm{Cu}(100)$ & substrate \\
\hline $\mathrm{Cu}$ & buffer \\
\hline $\mathrm{Cu}$ & buffer \\
\hline $\mathrm{Cu}$ & buffer \\
\hline $\left.\begin{array}{l}\mathrm{Cu}_{0.9} \mathrm{Co}_{0.1} \\
\mathrm{Cu}_{0.2} \mathrm{Co}_{0.8} \\
\mathrm{Cu}_{0.9} \mathrm{Co}_{0.1}\end{array}\right\}$ & {$[B]$} \\
\hline $\mathrm{Cu}$ & spacer \\
\hline$\vdots$ & \\
\hline $\mathrm{Cu}$ & spacer \\
\hline $\left.\begin{array}{l}\mathrm{Cu}_{0.9} \mathrm{Co}_{0.1} \\
\mathrm{Cu}_{0.2} \mathrm{Co}_{0.8} \\
\mathrm{Cu}_{0.9} \mathrm{Co}_{0.1}\end{array}\right\}$ & {$[B]$} \\
\hline $\mathrm{Cu}$ & buffer \\
\hline $\mathrm{Cu}$ & buffer \\
\hline $\mathrm{Cu}$ & buffer \\
\hline $\mathrm{Cu}(100)$ & substrate \\
\hline
\end{tabular}

When one applies the magnetic force theorem, the differences in total energies $E_{\mathrm{t}}$ are usually replaced by those of the band energies $E_{\mathrm{b}}$ [12]. Equation (10) implies that in a typical trilayer system, such as two slabs of Co separated by a $\mathrm{Cu}$ spacer, the orientation of the magnetization is rotated around $\widehat{\mathbf{n}}$ by a particular angle $\varphi$ in only one of the two magnetic slabs.

\section{Results and discussion}

Here we present the results of our calculations of CIP resistivities and GMR for a $\mathrm{Co} / \mathrm{Cu}_{N} / \mathrm{Co}$ trilayer with interdiffused interfaces for two characteristic thicknesses of the spacer (three and six layers of $\mathrm{Cu}$ ) inserted in semiinfinite $\mathrm{Cu}(100)$. The profile of the interdiffused interface - $[B] \mathrm{Cu}_{N}[B]$ - we have used is shown in Table 1. For the same systems we have also calculated the corresponding IEC. We will be interested in the functional behavior of $\rho_{x x}(\widehat{\mathbf{n}}, \varphi)$ and the corresponding $\operatorname{GMR}(\widehat{\mathbf{n}}, \varphi)$ as well as a comparison of the $\operatorname{IEC}(\widehat{\mathbf{n}}, \varphi)$ for three and six spacer layers, because for three layers of $\mathrm{Cu}$ the IEC is ferromagnetic, while for six layers it is close to the transition but within the antiferromagnetic regime.

All calculations were performed by using selfconsistent potentials obtained in terms of the fullyrelativistic spin-polarized SKKR method within the atomic sphere approximation, which served also for the determination of the IEC and electrical transport properties.

In Figure 1 the CIP resistivity $\rho_{x x}$ and GMR corresponding to three $\mathrm{Cu}$-spacer layers are shown for two different cases: (a) when the orientation of the magnetization is out of the plane of the layers, and (b) when it is in the plane. In the first case the orientation of the magnetization in one of the magnetic slabs $[B]$ is varied from $\widehat{\mathbf{z}}$ (parallel or "ferromagnetic" to the magnetization of the other slab, but perpendicular to the plane of the layers ) to $-\widehat{\mathbf{z}}$ (antiparallel or "antiferromagnetic"), while in the second case the change in orientation is from $\widehat{\mathbf{x}}$ to $-\widehat{\mathbf{x}}$ with the magnetization remaining in the plane of the layers. In this figure and in the following two figures, the actual calculated values are shown together with the two term fits found by using equation (8). For the GMR (lower half) these two terms are displayed as dashed and dotted lines. As one can see from Figure 1, as well as from Table 2 in which all the fitting parameters are listed, for three $\mathrm{Cu}$-spacer layers the GMR is essentially characterized by the first term in the expansion in equation (8). We have not found significant differences in the CIP resistivities whether we rotate the magnetization in or out of the plane, and conclude that the anisotropic magnetoresistance (AMR) is negligible for these trilayers.

For six Cu-spacer layers, we consider only rotations about the $\widehat{\mathbf{y}}$-axis. In Figure 2 we show the CIP resistivity $\rho_{x x}$ and GMR as the magnetization in one of the magnetic slabs $[B]$ goes from parallel (orientation of the magnetization perpendicular to the planes of atoms) to antiparallel. As can be seen from Table 2 and from a comparison to the system with three spacer layers, the first fitting parameter, $a_{1}=a_{1}(\widehat{\mathbf{y}})$, is reduced as we go from three to six spacer layers, which reflects the fall-off of the GMR with respect to the number of spacer layers, while the second fitting parameter, $a_{2}=a_{2}(\widehat{\mathbf{y}})$, is more or less unchanged by increasing the thickness of the spacer.

Completely different results are obtained for the functional behavior of the IEC for three and six $\mathrm{Cu}$-spacer layers; see Figure 3. We only considered rotations of the magnetization about the $\widehat{\mathbf{y}}$-axis. For three spacer layers ("ferromagnetic regime"), the IEC follows closely a fit essentially governed by the first term, while for six spacer layers ("antiferromagnetic regime"), $a_{1}$ is negative, as it should be, and very small. However, in this case the second fitting parameter is positive and considerably larger than the first one, i.e., for six spacer layers, the IEC viewed as a function of the rotation angle is mainly determined by the contribution from the second term. This result is interesting inasmuch as the value of the IEC for six spacer layers is close to the crossover from antiferromagnetic to ferromagnetic coupling and its magnitude (IEC $N^{2}$ ) is very small, e.g., compared to the case of three spacer layers. In the limit when the first - bilinear $\left(a_{1}\right)$ - term is close to zero, the second - biquadratic $\left(a_{2}\right)$ - term can dominate. It has been suggested that interface disorder (see Ref. [13]) and spin-orbit coupling can serve as possible causes for $\frac{\pi}{2}$-biquadratic coupling. Since we have used our fully-relativistic spin-polarized results to fit the angular dependence of the IEC, one possible interpretation of the contributions in the expansion equation (8) is that the first term refers to a rotation in spin space (no coupling to lattice), while in principle all higher terms reflect relativistic effects, which couple spin space to the lattice. If a two-term fit is sufficiently accurate, this implies that the biggest deviation from the bilinear contribution arises from the 

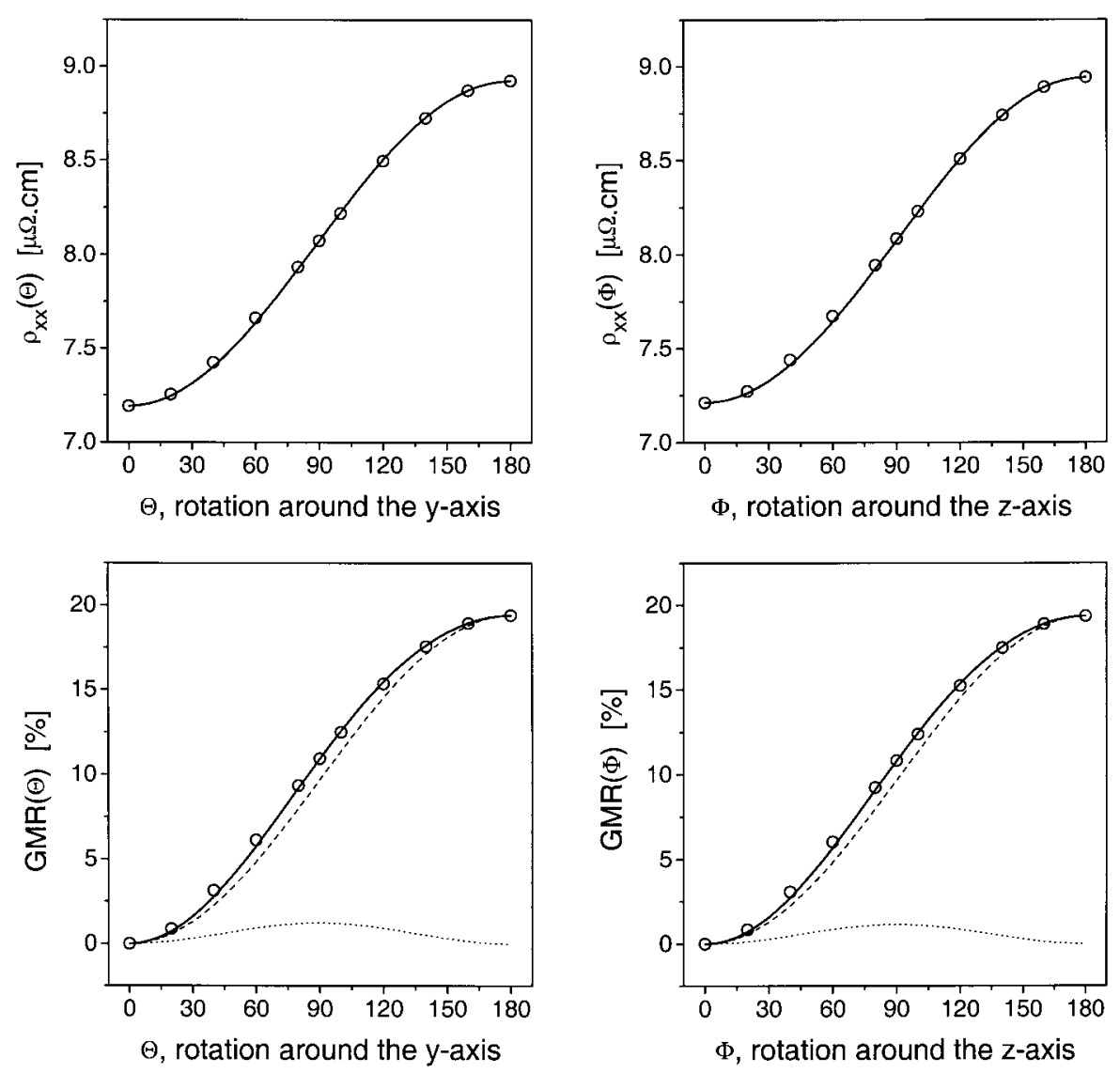

Fig. 1. CIP resistivity and MR for the trilayer system with three Cu-spacer layers and interdiffused interfaces. The figures on the left hand side are for the magnetization rotated out of the plane of the layers; the right hand side are for the magnetization rotated in plane. Circles represent calculated values, solid lines are the result of a two term fit according to equation (8), dashed and dotted lines display the first and second term respectively.

Table 2. Fitting parameters for the interdiffused trilayers $[B] \mathrm{Cu}_{N}[B]$.

\begin{tabular}{cccccc}
\hline & & \multicolumn{2}{c}{$N=3$} & \multicolumn{2}{c}{$N=6$} \\
quantity & unit & $a_{1}$ & $a_{2}$ & $a_{1}$ & $a_{2}$ \\
\hline$\rho_{x x}(\Theta)$ & {$[\mu \Omega \mathrm{cm}]$} & 0.868 & 0.018 & 0.195 & 0.037 \\
$\operatorname{GMR}(\Theta)$ & {$[\%]$} & 9.700 & 1.230 & 4.335 & 1.005 \\
& & & & & \\
$\operatorname{IEC}(\Theta)$ & {$[\mathrm{meV}]$} & 37.677 & -2.325 & -0.024 & 0.152 \\
\hline
\end{tabular}

biquadratic which favors a perpendicular arrangement of the magnetization in the two magnetic slabs of a trilayer system.

Clearly the CIP-MR and the IEC have different angular dependencies. It is interesting to observe that the angular dependence of the GMR for CIP seems to be governed mostly by the first term in the expansion, whereas - depending on the regime - in the case of the IEC the second term can indeed be important. For CPP, it has been proposed that in the asymptotic regime, i.e., when the number of spacer layers becomes very large, the oscillatory behavior of the IEC and CPP-MR with respect to the spacer thickness are closely related to the Fermi surface of the spacer material [6].

\section{Conclusion}

We have determined the functional dependence of the GMR for the CIP geometry and the IEC with respect to the relative angle between the orientations of the magnetization in the two magnetic slabs in a trilayer system. We find practically the same CIP resistivities whether we rotate the magnetization in or out of the plane of the layers; this leads us to conclude that the AMR is small for the trilayers we have studied. Only under certain conditions does the CIP-MR follow the same functional dependence as the IEC; as a general rule it does not. Interpreting the calculated results by means of a power series in the cosine of the relative angle for the GMR shows that the 

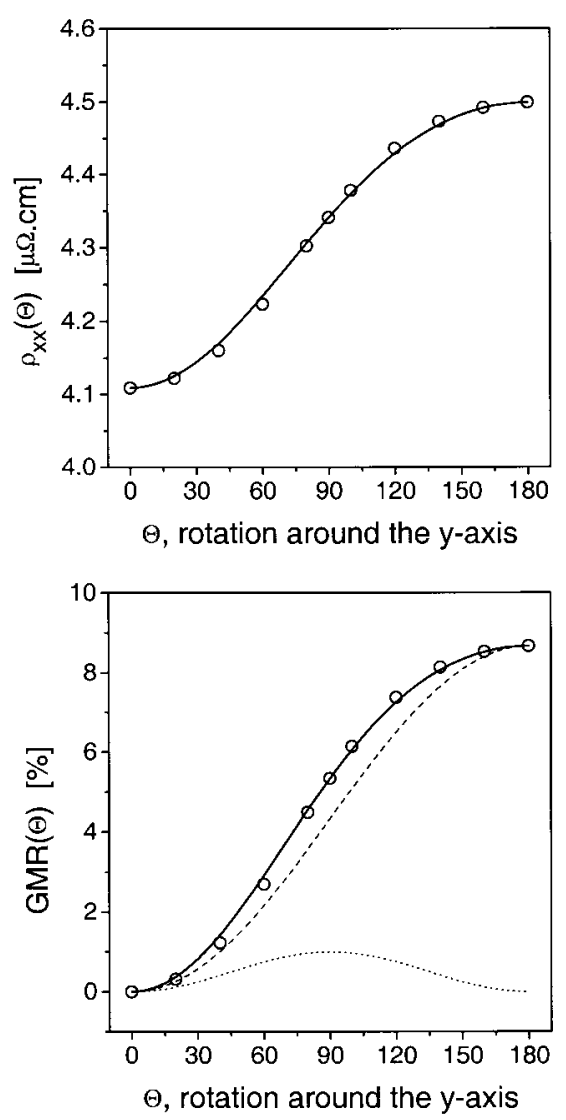

Fig. 2. CIP resistivity and MR for the trilayer system with six $\mathrm{Cu}$-spacer layers and interdiffused interfaces; the magnetization is rotated out of the plane of the layers. Circles represent calculated values, solid lines are the result of a two term fit according to equation (8), dashed and dotted lines display the first and second term respectively.

first coefficient falls off with the inverse of the number of layers, while the much smaller second coefficient seems to remain constant. Quite obviously, this is in contrast to the IEC, for which the first coefficient oscillates as a function of the spacer layer thickness, so that it passes through zero in order to change sign. At these nodes the second term in the power series dominates.

The present calculations were limited to trilayers with rather small layer thicknesses which can be realized at this time. However, they show that for systems in which we have only "normal" GMR, such as $\mathrm{Co} / \mathrm{Cu} / \mathrm{Co}$, (as contrasted to the inverse GMR effect [14]), the largest GMR is associated with the difference in the resistivities between the parallel and antiparallel configurations of the magnetizations of the magnetic layers; any perpendicular or other non-collinear configuration as a means of increasing the GMR can be ruled out.

Financial support from the Austrian Science Foundation (FWF P11626-PHY) and from the Austrian BMWV (AKTION WTZ-Österreich-Tschechien I.23) is gratefully acknowledged. This paper benefited also from a collaboration within the TMR Network on "Ab initio calculations of magnetic
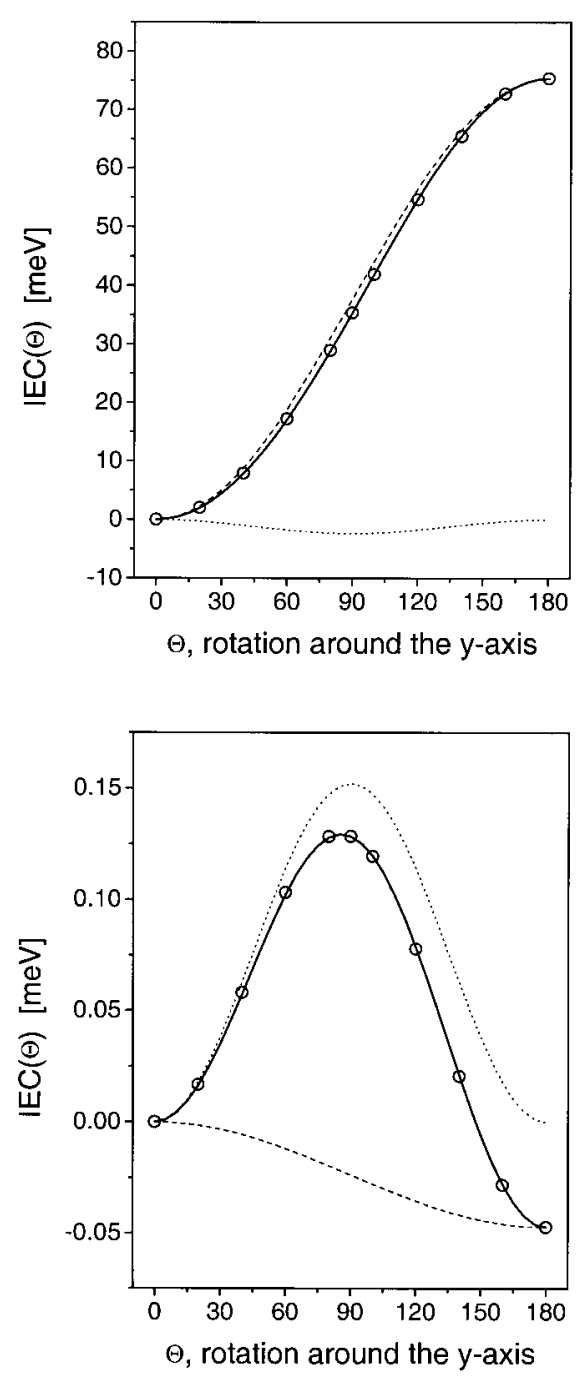

Fig. 3. IEC for the trilayer system with three (top) and six (bottom) Cu-spacer layers and interdiffused interfaces. Circles represent the calculated values, solid lines are the result of a two term fit according to equation (8), dashed and dotted lines display the first and second term respectively.

properties of surfaces, interfaces and multilayers" (Contract No. ERB4061PL951423). Two of us (JK and VD) also acknowledge the financial support from the Grant Agency of the Academy of Sciences of the Czech Republic (Project A1010829). The work by PML is supported by the Office of Naval Research (N00014-96-1-0203), together with the Defense Advanced Research Projects Agency (N00014-96-1-1207), the National Science Foundation (INT-9602192), and NATO (CRG 960340). We would like to thank the CNRS IDRIS computing center for the use of their T3E Cray.

\section{References}

1. S. Zhang, P.M. Levy, A. Fert, Phys. Rev. B 45, 8689 (1992).

2. A. Vedyayev, B. Dieny, N. Ryzhanova, J.B. Genin, C. Cowache, Europhys. Lett. 25, 465 (1994); A. Vedyayev, B. Dieny, N. Ryzhanova, J.B. Genin, Phys. Lett. A 185, 
117 (1994); A. Vedyayev, N. Ryzhanova, B. Dieny, P. Dauguet, P. Gandit, J. Chaussy, Phys. Rev. B 55, 3728 (1997); K. Wang, S. Zhang, P. M. Levy, Phys. Rev. B 54, 11965 (1996).

3. L.B. Steren, A. Barthelemy, J.L. Duvail, A. Fert, R. Morel, F. Petroff, P. Holody, R. Loloee, P.A. Schroeder, Phys. Rev. B 51, 292 (1995).

4. P. Dauguet, P. Gandit, J. Chaussy, S.F. Lee, A. Fert, P. Holody, Phys. Rev. B 54, 1083 (1996); B. Dieny, C. Cowache, A. Nossov, P. Dauguet, J. Chaussy, P. Gandit, J. Appl. Phys. 79, 6370 (1996).

5. C. Blaas, P. Weinberger, L. Szunyogh, P.M. Levy, C.B. Sommers, I. Mertig, Philos. Mag. B 78, 549 (1998); C. Blaas et al. (to be published).

6. J. Mathon, A. Umerski, M. Villeret, Phys. Rev. B 55, 14378 (1997).

7. J. Banhart, Philos. Mag. B 77, 85 (1998); Philos. Mag. B 77, 105 (1998).
8. P. Cortona, S. Doniach, C. Sommers, Phys. Rev. A 31, 2842 (1985); P. Weinberger, Electron Scattering Theory for Ordered and Disordered Matter (Clarendon, Oxford, 1990).

9. P. Weinberger, Philos. Mag. B 75, 509 (1997).

10. P. Weinberger, P.M. Levy, J. Banhart, L. Szunyogh, B. Újfalussy, J. Phys.-Cond. 8, 7677 (1996).

11. P. Weinberger, C. Sommers, U. Pustogowa, L. Szunyogh, B. Újfalussy, J. Phys. I France 7, 1299 (1997).

12. A. Oswald, R. Zeller, P.J. Braspenning, P.H. Dederichs, J. Phys. F: Met. Phys. 15, 193 (1985); A.I. Liechtenstein, M.I. Katsnelson, V.P. Antropor, V.A. Gubanov, J. Magn. Magn. Mat. 67, 65 (1987).

13. J.C. Slonczewski, Phys. Rev. Lett. 67, 3172 (1991); J. Magn. Magn. Mat. 126, 374 (1993); ibid. 150, 13 (1995).

14. S.Y. Hsu, A. Barthelemy, P. Holody, R. Loloee, P.A. Schroeder, A. Fert, Phys. Rev. Lett. 78, 2652 (1997); C. Vouille, A. Fert, A. Barhelemy, S.Y. Hsu, R. Loloee, P.A. Schroeder, J. Appl. Phys. 81, 4573 (1997). 